\title{
A Fulton-Hansen theorem for almost homogeneous spaces
}

\author{
János Kollár ${ }^{1} \cdot$ Aaron Landesman ${ }^{2}$ \\ Dedicated to Fabrizio Catanese on his 70th birthday \\ Received: 8 October 2020 / Accepted: 3 July 2021 / Published online: 15 July 2021 \\ (c) The Author(s) 2021
}

\begin{abstract}
We prove a generalization of the Fulton-Hansen connectedness theorem, where $\mathbb{P}^{n}$ is replaced by a normal variety on which an algebraic group acts with a dense orbit.
\end{abstract}

The product version of the Fulton-Hansen theorem [6] says that if $Y_{1}, Y_{2}$ are irreducible, projective varieties, $Y_{i} \rightarrow \mathbb{P}^{n}$ are finite morphisms and $\operatorname{dim} Y_{1}+\operatorname{dim} Y_{2}>\operatorname{dim} \mathbb{P}^{n}$, then $Y_{1} \times \mathbb{P}^{n} Y_{2}$ is connected. As Catanese emphasized both in the panoramic survey [3] and in the shorter version [2], one should look at the topological side of every question in algebraic geometry. Thus one should view the Fulton-Hansen theorem as a generalization of Lefschetz's hyperplane section theorem for the fundamental group. In this form, the quasiprojective version says that if $Y_{1}, Y_{2} \subset \mathbb{P}^{n}$ are normal, irreducible, quasi-projective varieties, $p_{i}: Y_{i} \rightarrow \mathbb{P}^{n}$ are quasi-finite morphisms, and $\operatorname{dim} Y_{1}+\operatorname{dim} Y_{2}>\operatorname{dim} \mathbb{P}^{n}$, then

$$
\pi_{1}\left(Y_{1} \times \mathbb{P}^{n}, g_{1}, g_{2} Y_{2}\right) \rightarrow \pi_{1}\left(Y_{1}\right) \times \pi_{1}\left(Y_{2}\right) \text { is surjective }
$$

for general $g_{1}, g_{2} \in \operatorname{Aut}\left(\mathbb{P}^{n}\right)$; here our notation means that we first compose $p_{i}$ with $g_{i}$ and then take fiber product. See [4,6] and [7, Remark 9.3] for the original proofs and Corollaries 23 for further discussion. Also see [18, Theorem 3.3.6] and [12, Theoreme 7.2].

Our main result is Theorem 2, which generalizes the above results. We replace $\left(\mathrm{PGL}_{n+1}, \mathbb{P}^{n}\right)$ by a pair $(G, X)$, where $X$ is a normal quasi-projective variety and $G$ is an algebraic group acting on $X$ with a fixed point and a dense open orbit. We obtain the Fulton-Hansen theorem by applying Theorem 2 to $X=\mathbb{P}^{n}$ and $G \subset \mathrm{PGL}_{n+1}$ the stabilizer of a point; see Corollary 2. This also implies the original form of the Fulton-Hansen theorem, which begins with a map $Z \rightarrow \mathbb{P}^{n} \times \mathbb{P}^{n}$ and shows that the preimage of the diagonal is connected, see Corollary 3.

The usual approaches to Fulton-Hansen-type theorems exploit ampleness properties of the tangent bundle $T_{X}$. We get the strongest result when $T_{X}$ is ample, which holds only for $X=\mathbb{P}^{n}$ [20]. For other homogeneous spaces $X=G / P$, one can develop a theory that

Aaron Landesman

aaronlandesman@stanford.edu

János Kollár

kollar@math.princeton.edu

1 Princeton University, Princeton, NJ 08544-1000, USA

2 Stanford University, Stanford, CA 94305, USA 
measures the failure of ampleness $[5,7,9,21,22]$. This leads to results that give $\pi_{1}$-surjectivity if $\operatorname{dim} Y_{1}+\operatorname{dim} Y_{2}$ is large enough. For example, let $0 \leq r \leq n / 2$ and let $X=\operatorname{Gr}\left(\mathbb{P}^{r}, \mathbb{P}^{n}\right)$. By [9, Connectedness Theorem, p. 361] the map $\pi_{1}\left(Y_{1} \cap Y_{2}\right) \rightarrow \pi_{1}\left(Y_{1}\right) \times \pi_{1}\left(Y_{2}\right)$ is surjective provided

$$
\operatorname{dim} Y_{1}+\operatorname{dim} Y_{2}>\operatorname{dim} X+\operatorname{dim} \operatorname{Gr}\left(\mathbb{P}^{r-1}, \mathbb{P}^{n-2}\right) .
$$

Here $\operatorname{dim} X$ is what we expect on the right hand side of $(*)$ from the naive dimension count. For $r=0, \operatorname{dim} \operatorname{Gr}\left(\mathbb{P}^{r-1}, \mathbb{P}^{n-2}\right)=0$, and we recover the Fulton-Hansen theorem. However, for $r \gg 0$ the extra term gets quite large. Nonetheless, this bound is optimal, see Example 8 .

Our aim is to prove that, for many group actions, the naive dimension estimate $\operatorname{dim} Y_{1}+$ $\operatorname{dim} Y_{2}>\operatorname{dim} X$ is enough to give $\pi_{1}$-surjectivity, unless the intersection $Y_{1} \cap Y_{2}$ is very degenerate.

Notation 1 We work over an algebraically closed field $k$ unless otherwise specified. Let $G$ be a connected algebraic group acting on a normal $k$-variety $X$. We usually assume that the action is faithful.

The action is denoted by $(g, x) \mapsto g x$. If $p: Y \rightarrow X$ is a morphism then it is convenient to use the shorthand $g Y \rightarrow X$ for $g \circ p: Y \rightarrow X$.

We consider the case when the $G$-action has a dense open orbit, denoted by $X^{\circ}$, and also a fixed point $x_{0} \in X$. For any $n \geq 0, G$ acts linearly on the $n$th order infinitesimal neighborhood $\mathscr{O}_{X, x_{0}} / \mathfrak{m}_{x_{0}}^{n}$, where $\left(\mathscr{O}_{X, x_{0}}, \mathfrak{m}_{x_{0}}\right)$ is the local ring at $x_{0}$ and its maximal ideal. If we assume the action of $G$ on $X$ is faithful, this induced action is also faithful for sufficiently large $n$, and so, in this case, $G$ is a linear algebraic group.

We use $\pi_{1}\left(Z, z_{0}\right)$ to denote the topological fundamental group of $Z(\mathbb{C})$ if we are over $\mathbb{C}$ and the étale fundamental group of $Z$ otherwise. We will usually include basepoints, but sometimes omit them when it is not important to keep track of them.

The main result is easier to state for normal subvarieties over $\mathbb{C}$. We prove a more general version in Theorem 2, where the characteristic is arbitrary, the stabilizer may be disconnected and the $Y_{i}$ are not necessarily subvarieties.

Theorem 1 Let $G$ be a connected algebraic group acting on a normal variety $X$ defined over $\mathbb{C}$. Assume that the action has a fixed point $x_{0} \in X$ and a dense open orbit $X^{\circ} \subset X$ such that the stabilizer $H \subset G$ of any point in $X^{\circ}$ is connected. Let $Y_{1}, Y_{2}$ be irreducible, normal, locally closed subvarieties of $X$ containing $x_{0}$. Assume that

(1) $\operatorname{dim} Y_{1}+\operatorname{dim} Y_{2}>\operatorname{dim} X$ and

(2) there is an irreducible component $Z \subset Y_{1} \cap Y_{2}$ of the expected dimension $\operatorname{dim} Y_{1}+$ $\operatorname{dim} Y_{2}-\operatorname{dim} X$, that contains $x_{0}$ and is not disjoint from $X^{\circ}$.

Then,

$$
\pi_{1}\left(g_{1} Y_{1} \cap g_{2} Y_{2}, x_{0}\right) \rightarrow \pi_{1}\left(Y_{1}, x_{0}\right) \times \pi_{1}\left(Y_{2}, x_{0}\right) \text { is surjective }
$$

for general $\left(g_{1}, g_{2}\right) \in G \times G$.

Note that the conclusion of Theorem 1 is pretty much the strongest that one can hope for: If $Y_{1} \cap Y_{2}$ is disjoint from $X^{\circ}$, then $g_{1} Y_{1} \cap g_{2} Y_{2}$ is never in 'general position,' and if an irreducible component does not contain the base point $x_{0}$, then its contribution to the fundamental group is hard to control; see also Examples 7-8. For counterexamples with disconnected stabilizers see Examples 10-11.

We next state and prove Theorem 2, from which Theorem 1 follows. We then derive generalizations of the Fulton-Hansen theorem and further applications. We conclude with various examples and counterexamples. 


\section{Proof of the main theorem}

We start with some preliminary lemmas.

Lemma 1 Let $f: U \rightarrow V$ be a dominant morphism of irreducible varieties and $\sigma: V \rightarrow U$ a section. Then $U_{v}$ is connected for general $v \in V$.

Proof It is enough to show that the geometric generic fiber of $f$ is connected. By irreducibility of $U$, the generic fiber of $f$ is irreducible. The generic fiber may fail to be geometrically irreducible, but all geometric components must pass through the image of $\sigma$, and hence the geometric generic fiber is connected.

We also require the following slight variant of [14, Theorem 2], which follows from [14, Theorem 2] and upper semicontinuity of dimension of fibers.

Lemma 2 Let $G$ be a connected algebraic group acting transitively on a normal $k$-variety $X$. Let $Y_{1}$ and $Y_{2}$ be irreducible, normal varieties and $p_{i}: Y_{i} \rightarrow X$ quasi-finite morphisms. Then there is a dense open subset $U \subset G \times G$ such for all $\left(g_{1}, g_{2}\right) \in U, g_{1} Y_{1} \times{ }_{X} g_{2} Y_{2}$ is

(1) either empty

(2) or of pure dimension $\operatorname{dim} Y_{1}+\operatorname{dim} Y_{2}-\operatorname{dim} X$

Moreover, if $g_{1} Y_{1} \times_{X} g_{2} Y_{2}$ is non-empty and of dimension $\leq \operatorname{dim} Y_{1}+\operatorname{dim} Y_{2}-\operatorname{dim} X$ for some $\left(g_{1}, g_{2}\right) \in G \times G$, then (2) holds.

Notation 2 Let $X$ be a $k$-variety, $x_{0} \in X$ a closed point and $X^{\circ} \subset X$ a dense open subset. Let $p: Z \rightarrow X$ be a quasi-finite morphism. We let $Z^{\circ}$ denote $p^{-1}\left(X^{\circ}\right)$. For $z_{0} \in p^{-1}\left(x_{0}\right)$, let $Z\left(z_{0}\right) \subset Z$ denote the union of those irreducible components that contain $z_{0}$ and whose images are not disjoint from $X^{\circ}$. We say that $z_{0}$ is a good limit point of $Z$ if $Z\left(z_{0}\right) \neq \emptyset$.

Lemma 3 Let $G$ be a connected algebraic group acting on a normal variety $X$ with a fixed point $x_{0} \in X$ and a dense open orbit $X^{\circ}$ with stabilizer $H \subset G$. Let $H_{0} \subset H$ denote the identity component of $H$.

Let $Y_{1}$ and $Y_{2}$ be irreducible, normal varieties and $p_{i}: Y_{i} \rightarrow X$ quasi-finite morphisms such that $Y_{i}^{\circ}:=p_{i}^{-1}\left(X^{\circ}\right)$ are nonempty.

Let $I \subset G \times G \times Y_{1} \times Y_{2}$ parametrize quadruples $\left(g_{1}, g_{2}, y_{1}^{\prime}, y_{2}^{\prime}\right)$ such that $g_{1}^{-1} p_{1}\left(y_{1}^{\prime}\right)=$ $g_{2}^{-1} p_{2}\left(y_{2}^{\prime}\right)$. Let $I^{\circ} \subset I$ be the preimage of $Y_{1}^{\circ} \times Y_{2}^{\circ}$ and $\bar{I}^{\circ} \subset I$ its closure. We have

$$
G \times G \stackrel{\pi}{\leftarrow} \bar{I}^{\circ} \stackrel{\tau}{\rightarrow} Y_{1} \times Y_{2} .
$$

Then

(2) $I^{\circ} \rightarrow Y_{1}^{\circ} \times Y_{2}^{\circ}$ is a locally trivial $G \times H$-bundle.

(3) $I^{\circ}$ has at most $\left|H / H_{0}\right|$ irreducible components.

(4) Every good limit point $z_{0}=\left(y_{1}, y_{2}\right)$ lying on a component of $Y_{1} \times_{X} Y_{2}$ of dimension $\operatorname{dim} Y_{1}+\operatorname{dim} Y_{2}-\operatorname{dim} X$ gives a rational section $\pi_{z_{0}}:\left(g_{1}, g_{2}\right) \mapsto\left(g_{1}, g_{2}, y_{1}, y_{2}\right)$.

Let $j$ index those irreducible components $\bar{I}^{\circ}\left(z_{0}, j\right) \subset \bar{I}^{\circ}$ that contain the image of $\pi_{z_{0}}$. Then the following hold.

(5) For any $j$ as above, the image of $\pi_{1}\left(\bar{I}^{\circ}\left(z_{0}, j\right)\right) \rightarrow \pi_{1}\left(Y_{1} \times Y_{2}\right)$ has index $\leq\left|H / H_{0}\right|$.

(6) $\bar{I}^{\circ}\left(z_{0}, j\right) \cap \pi^{-1}\left(g_{1}, g_{2}\right)$ is a nonempty union of irreducible components of $\left(g_{1} Y_{1} \times_{X}\right.$ $\left.g_{2} Y_{2}\right)\left(z_{0}\right)$ for general $\left(g_{1}, g_{2}\right) \in G \times G$. 
(7) If $H$ is connected then $\pi^{-1}\left(g_{1}, g_{2}\right)$ is connected for general $\left(g_{1}, g_{2}\right) \in G \times G$.

Remark 3.8. It can happen that $g_{1} Y_{1} \times{ }_{X} g_{2} Y_{2}$ is always reducible, though this seems to be rare; see Example 4.

Proof Claims (2) and (4) are clear and (2) implies (3). There is nothing further to prove if there are no good limit points.

Otherwise let $z_{0}=\left(y_{1}, y_{2}\right)$ be a good limit point. Set $K=k(G \times G)$. We next prove (6). By Lemma 2, the generic fiber $\bar{I}^{\circ}\left(z_{0}, j\right)_{K}$ of the projection $\bar{I}^{\circ}\left(z_{0}, j\right) \rightarrow G \times G$ is nonempty, hence irreducible over $K$, and it has a $K$-point, given by $z_{0}$. Note there is some such component $\bar{I}^{\circ}\left(z_{0}, j\right)$ because $z_{0}$ is a good limit point. The union of those geometric irreducible components of $\bar{I}^{\circ}\left(z_{0}, j\right)_{K}$ that contain this $K$-point is defined over an extension of $K$. Thus, every geometric irreducible component of $\bar{I}^{\circ}\left(z_{0}, j\right)_{K}$ contains this $K$-point. Hence, for general $\left(g_{1}, g_{2}\right) \in G \times G$, every irreducible component of $\bar{I}^{\circ}\left(z_{0}, j\right) \cap \pi^{-1}\left(g_{1}, g_{2}\right)$ contains $\left(g_{1}, g_{2}, z_{0}\right)$, proving (6).

As for (5), $I^{\circ} \rightarrow Y_{1}^{\circ} \times Y_{2}^{\circ}$ factors as

$$
I^{\circ} \stackrel{\tau_{1}}{\rightarrow} I^{\circ} /\left(G \times H_{0}\right) \stackrel{\tau_{2}}{\rightarrow} Y_{1}^{\circ} \times Y_{2}^{\circ} .
$$

Here $\tau_{1}$ is a principal $G \times H_{0}$-bundle with connected fibers and $\tau_{2}$ is finite, étale of degree $\left|H / H_{0}\right|$ (but possibly disconnected). Thus the image of

$$
\pi_{1}\left(I^{\circ}\right) \rightarrow \pi_{1}\left(Y_{1}^{\circ} \times Y_{2}^{\circ}\right)
$$

is a subgroup of index $\leq\left|H / H_{0}\right|$. Observe that $\pi_{1}\left(Y_{1}^{\circ} \times Y_{2}^{\circ}\right) \rightarrow \pi_{1}\left(Y_{1} \times Y_{2}\right)$ is surjective as $Y_{1}$ and $Y_{2}$ are normal; see [7, 0.7.B]. We can thus factor $\tau: \bar{I}^{\circ}\left(z_{0}, j\right) \rightarrow Y_{1} \times Y_{2}$ as

$$
\bar{I}^{\circ}\left(z_{0}, j\right) \stackrel{\tau_{0}}{\rightarrow}\left(Y_{1} \times Y_{2}\right)^{\sim} \stackrel{\tilde{\tau}}{\rightarrow} Y_{1} \times Y_{2},
$$

where $\tilde{\tau}$ is finite, étale of degree $\leq\left|H / H_{0}\right|$ and

$$
\pi_{1}\left(\bar{I}^{\circ}\left(z_{0}, j\right)\right) \rightarrow \pi_{1}\left(\left(Y_{1} \times Y_{2}\right)^{\sim}\right) \text { is surective. }
$$

This proves (5) and (7) follows from Lemma 1.

The proof of Theorem 2 will follow from the above, together with the following important fact from topology.

Remark 1 Suppose we are given a map $f: X \rightarrow Y$ of varieties over $\mathbb{C}$. We claim that there is a Zariski open subset $U \subset Y$ such that $X \times_{Y} U \rightarrow U$ is locally a trivial bundle in the Euclidean topology. More precisely, for any point $p \in U^{\text {an }}$, the analytic space associated to $U$, there is an analytic open set $V \subset U^{\text {an }}$ with $p \in V$ and a homeomorphism of topological spaces spaces $\left(f^{\text {an }}\right)^{-1}(V) \simeq\left(f^{\text {an }}\right)^{-1}(p) \times V$ over $V$.

The claim above follows from [8, Part I, Theorem 1.7, p. 43] and its proof, although it is not explicitly stated there, so we comment on the details. First, [11, §4, Theorem 1] shows that the pieces in the stratification of [8, Part I, Theorem 1.7, p. 43] can be taken to be complex analytic. (Note that the pieces of the stratification are not necessarily real analytic when the map is real analytic, see [8, Caution, p. 43].) To prove the existence of the desired Zariski open set $U \subset Y$, one may work with proper compactifications $\bar{X}, \bar{Y}$ of $X$ and $Y$, which exist by Nagata's compactification theorem. We may then use the last sentence of [8, Part I, Theorem 1.7, p. 43] to ensure the boundaries of these closures are also unions of strata of the stratifications. Hence, we also obtain stratifications of the open parts $X$ and $Y$. Then, [8, Part I, §1.6, p. 43] explains why the resulting map is topologically trivial over pieces of 
the stratification. Finally, to show that one of the pieces of the stratification of $Y$ is Zariski open, we note that there is a piece whose complement is a complex analytic proper closed subvariety, hence algebraic by Chow's theorem.

$\mathbf{8}$ (Fundamental groups of fibers) Let $S, W, Z$ be irreducible $k$-varieties, $Z$ is normal. Assume that we have dominant morphisms

$$
S \stackrel{q}{\longleftarrow} W \stackrel{p}{\longrightarrow} Z
$$

and a section $\sigma: S \rightarrow W$ of $q$ such that $p \circ \sigma: S \rightarrow Z$ is a constant morphism with image $z_{0} \in Z$. Assume that $\pi_{1}(W) \rightarrow \pi_{1}(Z)$ is surjective and $W \rightarrow Z$ is a flat map with irreducible fibers. We aim to understand whether the induced homomorphism $\pi_{1}\left(W_{s}, \sigma(s)\right) \rightarrow \pi_{1}\left(Z, z_{0}\right)$ is surjective for general $s \in S$.

Case .1. $k=\mathbb{C}$. By Remark 1, there is an open, dense subset $S^{\circ} \subset S$ such that $q$ is a topological fiber bundle over $S^{\circ}$. Set $W^{\circ}:=q^{-1}\left(S^{\circ}\right)$. Note that $W^{\circ} \rightarrow S^{\circ}$ has a section that gets contracted by $p$. Thus

$$
\pi_{1}\left(W_{s}, \sigma(s)\right) \rightarrow \pi_{1}\left(Z, z_{0}\right) \text { is surjective for every } s \in S^{\circ} .
$$

Case .2. char $k=0$. By the Lefschetz principle we get that (.1.a) holds for the étale fundamental group.

Case .3. char $k>0$. Let $\pi_{Z}:\left(Z^{\prime}, z_{0}^{\prime}\right) \rightarrow\left(Z, z_{0}\right)$ be an irreducible, finite, étale cover corresponding to a finite quotient $\pi_{1}\left(Z, z_{0}\right) \rightarrow H$. By pullback we obtain a connected, finite, étale cover $\pi_{W}: W^{\prime} \rightarrow W$. Here, $W^{\prime}$ is irreducible because $W^{\prime} \rightarrow Z^{\prime}$ is flat with irreducible fibers. Further, $\sigma$ lifts (non-uniquely) to a section $\sigma^{\prime}: S \rightarrow W^{\prime}$ such that $p^{\prime} \circ \sigma^{\prime}$ maps $S$ to $z_{0}^{\prime}$. Thus, by Lemma 1 , there is an open, dense subset $S_{H}^{\circ} \subset S$ such that $W_{s}^{\prime}$ is connected for $s \in S_{H}^{\circ}$. That is,

$$
\pi_{1}\left(W_{s}, \sigma(s)\right) \rightarrow \pi_{1}\left(Z, z_{0}\right) \rightarrow H \text { is surjective for every } s \in S_{H}^{\circ} .
$$

In this case we say that $\pi_{1}\left(W_{s}, \sigma(s)\right) \rightarrow \pi_{1}\left(Z, z_{0}\right)$ is surjective on finite quotients for general $s \in S$.

Remark .4 The complication in .3 is that $S_{H}^{\circ}$ depends on $H$, and, if char $k>0$, then $\cap_{H} S_{H}^{\circ}$ may be empty. That is, there may not be any closed point $s \in S$ for which $\pi_{1}\left(W_{s}, \sigma(s)\right) \rightarrow$ $\pi_{1}\left(Z, z_{0}\right)$ is surjective; see Example 9 .

Combining Lemma 3 with Paragraph, can now prove our main theorem.

Theorem 2 Let $G$ be a connected algebraic group acting on a normal $k$-variety $X$ with a fixed point $x_{0} \in X$ and a dense open orbit $X^{\circ}$ with stabilizer $H \subset G$. Let $\left(y_{1}, Y_{1}\right),\left(y_{2}, Y_{2}\right)$ be irreducible, normal, pointed varieties and $p_{i}: Y_{i} \rightarrow X$ quasi-finite morphisms such that $p_{i}\left(y_{i}\right)=x_{0}$. Assume that

(1) $1 \leq \operatorname{dim}\left(Y_{1}^{\circ} \times_{X} Y_{2}^{\circ}\right) \leq \operatorname{dim} Y_{1}+\operatorname{dim} Y_{2}-\operatorname{dim} X$, and

(2) $Y_{1} \times_{X} Y_{2}$ has a good limit point $z_{0}=\left(y_{1}, y_{2}\right)$, as in Notation 2. Then, for general $\left(g_{1}, g_{2}\right) \in G \times G$,

(3) the closure of $g_{1} Y_{1}^{\circ} \times{ }_{X} g_{2} Y_{2}^{\circ}$ in $g_{1} Y_{1} \times_{X} g_{2} Y_{2}$ has at most $\left|H / H_{0}\right|$ connected components, and

(4) there is a subgroup $\Gamma \subset \pi_{1}\left(Y_{1}, y_{1}\right) \times \pi_{1}\left(Y_{2}, y_{2}\right)$ of index at most $\left|H / H_{0}\right|$ such that the natural map,

$$
\pi_{1}\left(g_{1} Y_{1} \times{ }_{X} g_{2} Y_{2}, z_{0}\right) \rightarrow \Gamma
$$

is surjective if char $k=0$, and surjective on finite quotients if char $k>0$. 
Proof. Fix an index $j$ as in Lemma 3 and consider the diagram

$$
G \times G \stackrel{\pi}{\leftarrow} \bar{I}^{\circ}\left(z_{0}, j\right) \stackrel{\tau_{0}}{\rightarrow}\left(Y_{1} \times Y_{2}\right) \stackrel{\sim}{\rightarrow} Y_{1} \times Y_{2},
$$

whose right hand side is defined in (3.9). Set

$$
\Gamma:=\pi_{1}\left(\left(Y_{1} \times Y_{2}\right)^{\sim}\right) \subset \pi_{1}\left(Y_{1}, y_{1}\right) \times \pi_{1}\left(Y_{2}, y_{2}\right) .
$$

Next apply the discussions in Paragraph to conclude that

$$
\pi_{1}\left(\bar{I}^{\circ}\left(z_{0}, j\right) \cap \pi^{-1}\left(g_{1}, g_{2}\right)\right) \rightarrow \pi_{1}\left(\left(Y_{1} \times Y_{2}\right)^{\sim}\right)
$$

is surjective if char $k=0$, and surjective on finite quotients if char $k>0$.

\section{Fulton-Hansen-type theorems}

We get the following version of the Fulton-Hansen theorem.

Corollary 1 (Fulton-Hansen theorem I) Let $\mathcal{G}$ be a connected algebraic group acting 2transitively on a quasi-projective variety $X$. Let $Z$ be a normal, irreducible variety with $\operatorname{dim} Z>\operatorname{dim} X$ and $p: Z \rightarrow X \times X$ quasi-finite. Then, for general $\left(g_{1}, g_{2}\right) \in \mathcal{G} \times \mathcal{G}$, and $\Delta_{X}: X \rightarrow X \times X$ the diagonal map,

(1) $Z \times\left(g_{1}, g_{2}\right) \circ p, X \times X, \Delta_{X} X$ is connected and

(2) the natural map,

$$
\pi_{1}\left(Z \times{\left(g_{1}, g_{2}\right) \circ p, X \times X, \Delta_{X}} X, z_{0}\right) \rightarrow \pi_{1}(Z, z) \times \pi_{1}(X, x),
$$

is surjective if char $k=0$ and surjective on finite quotients if char $k>0$.

Proof (assuming Corollary 5). For general $\left(g_{1}, g_{2}\right) \in \mathcal{G} \times \mathcal{G}$ choose $z_{0}=(z, x) \in$ $Z \times\left(g_{1}, g_{2}\right) \circ p, X \times X, \Delta_{X} X$ and define $x_{0} \in X$ as the point such that $\left(x_{0}, x_{0}\right)=\left(\left(g_{1}, g_{2}\right) \circ p\right)(z)$. Let $G \subset \mathcal{G}$ denote the identity component of the stabilizer of $x_{0}$. Note that $G$ acts transitively on $X \backslash\left\{x_{0}\right\}$. (By Remark 2, the stabilizer in $\mathcal{G}$ of $x_{0}$ is already connected, though we will not need this fact.)

We aim to apply Theorem 2 to $G \times G$ acting on $X \times X$ with fixed point $\left(x_{0}, x_{0}\right)$ and dense open orbit $\left(X \backslash\left\{x_{0}\right\}\right) \times\left(X \backslash\left\{x_{0}\right\}\right)$. We now check the hypotheses. As an itinitial reduction, the statement for general translations of both $Z$ and $X$ is equivalent to the analogous one for only translates of $Z$ because $Z \times{ }_{\left(g_{1}, g_{2}\right) \circ p, X \times X,\left(h_{1}, h_{2}\right) \circ \Delta_{X}} X \simeq Z \times{ }_{\left(h_{1}^{-1} g_{1}, h_{2}^{-1} g_{2}\right) \circ p, X \times X, \Delta_{X}} X$. Now, note that, $\operatorname{dim}_{z_{0}}\left(Z \times{ }_{\left(g_{1}, g_{2}\right) \circ p, X \times X, \Delta_{X}} X\right)=\operatorname{dim} Z+\operatorname{dim} X-\operatorname{dim} X \times X>0$ by Lemma 2. Since $\Delta_{X}(X) \backslash\left\{\left(x_{0}, x_{0}\right)\right\}$ is contained in the dense orbit $\left(X \backslash\left\{x_{0}\right\}\right) \times\left(X \backslash\left\{x_{0}\right\}\right)$, $z_{0}$ is a good limit point of $Z \times{ }_{\left(g_{1}, g_{2}\right) \circ p, X \times X, \Delta_{X}} X$. Additionally, it follows from Corollary 5 that the stabilizer $H \subset G$ of the $G$ action on $X \backslash\left\{x_{0}\right\}$ is connected. Therefore, the stabilizer in $G \times G$ of a point of $X \backslash\left\{x_{0}\right\} \times X \backslash\left\{x_{0}\right\}$ is also connected.

We could have proven Corollary 1 using Remark 2 in place of Corollary 5. We opted to use the latter as it leads to a more self-contained proof.

An important special case of Corollary 1 is the the case where $Z=Y_{1} \times Y_{2}$ and $p=p_{1} \times p_{2}$ for $p_{i}: Y_{i} \rightarrow X$ quasi-finite morphisms. Because this is typically how Corollary 1 is applied, we now restate it in this case.

Corollary 2 (Fulton-Hansen theorem II) Let $\mathcal{G}$ be a connected algebraic group acting 2transitively on a quasi-projective variety $X$. Let $Y_{1}, Y_{2}$ be normal, irreducible varieties and 
$p_{i}: Y_{i} \rightarrow X$ quasi-finite morphisms. Assume that $\operatorname{dim} Y_{1}+\operatorname{dim} Y_{2}>\operatorname{dim} X$. Then, for general $\left(g_{1}, g_{2}\right) \in \mathcal{G} \times \mathcal{G}$,

(1) $g_{1} Y_{1} \times{ }_{X} g_{2} Y_{2}$ is connected and

(2) the natural map,

$$
\pi_{1}\left(g_{1} Y_{1} \times_{X} g_{2} Y_{2}, z_{0}\right) \rightarrow \pi_{1}\left(Y_{1}, y_{1}\right) \times \pi_{1}\left(Y_{2}, y_{2}\right),
$$

is surjective if char $k=0$ and surjective on finite quotients if char $k>0$.

If $X$ and $Z$ as in Corollary 1 are proper, then connectedness of $Z \times{\left(g_{1}, g_{2}\right) \circ p, X \times X, \Delta_{X} X \text { for }}$ general $\left(g_{1}, g_{2}\right) \in \mathcal{G} \times \mathcal{G}$ implies connectedness for every $\left(g_{1}, g_{2}\right)$. This is sometimes called the Enriques-Severi-Zariski connectedness principle, proved by combining Stein factorization with Zariski's main theorem.

Thus we recover the original setting of the Fulton-Hansen therem [6].

Corollary 3 (Fulton-Hansen theorem III) Let $\mathcal{G}$ be a connected algebraic group acting 2transitively on a projective variety $X$. Let $Z$ be a normal irreducible proper variety with $\operatorname{dim} Z>\operatorname{dim} X$ and $p: Z \rightarrow X \times X$ a finite morphism. Then $Z \times_{p, X \times X, \Delta_{X}} X$ is connected. In particular, if $Z=Y_{1} \times Y_{2}$ and $p=p_{1} \times p_{2}$ for $p_{i}: Y_{i} \rightarrow X$ finite morphisms, then $Y_{1} \times p_{1}, X, p_{2} Y_{2}$ is connected.

Remark 2 The most important example of a 2-transitive action is $\left(\mathrm{PGL}_{n+1}, \mathbb{P}^{n}\right)$,

The 2-transitive case seems much more general, but in fact there are very few such pairs $(\mathcal{G}, X)$. By [15] $\left(\mathrm{PGL}_{n+1}, \mathbb{P}^{n}\right)$ is the only pair with $X$ projective. The pairs with $X$ quasiprojective are all of the form $\left(G \ltimes \mathbb{G}_{a}^{n}, \mathbb{A}^{n}\right)$ where $G \subset \mathrm{GL}_{n}$ is a product $C \cdot \bar{G}$, for $C$ a subgroup of the central $\mathbb{G}_{m} \subset \mathrm{GL}_{n}$ and $\bar{G}$ is one of the following:

(1) $n=1, \bar{G}=\mathrm{GL}_{1}$,

(2) $n \geq 2, \bar{G}=\mathrm{SL}_{n}$,

(3) $n=2 m$ is even, $\bar{G}=\mathrm{Sp}_{m}$,

(4) $n=6$, the characteristic is 2 , and $\bar{G}=\mathrm{G}_{2}$.

(Note that $\mathrm{G}_{2}$ does not have a nontrivial 6-dimensional representation in characteristics $\neq 2$.)

There is, however, a very long list of pairs $(G, X)$ such that $G(\mathbb{R})$ acts 2-transitively on $X(\mathbb{R})$; see $[16,25]$. So the following variant applies in many more cases.

Corollary 4 Let $X$ be a variety defined over $\mathbb{R}$ and $G$ a connected algebraic group acting on it such that the $G(\mathbb{R})$ action on $X(\mathbb{R})$ is 2-transitive. Assume that for $x_{0} \neq x_{1} \in X(\mathbb{R})$, the stabilizer of the ordered pair $\left(x_{0}, x_{1}\right)$ is connected (over $\mathbb{C}$ ). Let $Z_{1}, Z_{2}$ be irreducible, normal varieties and $p_{i}: Z_{i} \rightarrow X$ quasi-finite morphisms. Assume that $\operatorname{dim} Z_{1}+\operatorname{dim} Z_{2}>\operatorname{dim} X$ and the $Z_{i}$ have smooth real points. Then,

$$
\pi_{1}\left(g_{1} Z_{1} \times_{X} g_{2} Z_{2}\right) \rightarrow \pi_{1}\left(Z_{1}\right) \times \pi_{1}\left(Z_{2}\right) \text { is surjective }
$$

for general $\left(g_{1}, g_{2}\right) \in G(\mathbb{R}) \times G(\mathbb{R})$ and for general $\left(g_{1}, g_{2}\right) \in G(\mathbb{C}) \times G(\mathbb{C})$.

There are also some non-transitive group actions for which we get a Fulton-Hansen-type result, with obvious exceptions.

Example 1 (Orthogonal group) Let $\mathrm{GO}_{q}:=\mathbb{G}_{m} \cdot \mathrm{O}_{q}$ be the group of orthogonal similitudes acting on the $n$-dimensional vector space $V^{n}$, where $q$ is a nondegenerate quadratic form. There are 3 orbits, $\{0\},(q=0) \backslash\{0\}$, and the dense open orbit is $V^{n} \backslash(q=0)$. 
Claim 1.1. Let $0 \in Y_{i} \subset \mathbb{A}^{n}$ be irreducible, normal, locally closed subvarieties. Assume that $Y_{i} \not \subset(q=0)$ and $\operatorname{dim} Y_{1}+\operatorname{dim} Y_{2}>n$. Then

$$
\pi_{1}\left(g_{1} Y_{1} \cap g_{2} Y_{2}, 0\right) \rightarrow \pi_{1}\left(Y_{1}, 0\right) \times \pi_{1}\left(Y_{2}, 0\right) \text { is surjective }
$$

for general $\left(g_{1}, g_{2}\right) \in \mathrm{GO}_{q} \times \mathrm{GO}_{q}$.

Proof Since $\mathbb{A}^{n}$ is smooth,

$$
\operatorname{dim}_{0}\left(g_{1} Y_{1} \cap g_{2} Y_{2}\right) \geq \operatorname{dim} Y_{1}+\operatorname{dim} Y_{2}-n .
$$

Thus we have a good limit point if

$$
\operatorname{dim}_{0}\left(g_{1} Y_{1} \cap g_{2} Y_{2} \cap(q=0)\right)<\operatorname{dim} Y_{1}+\operatorname{dim} Y_{2}-n .
$$

Since $Y_{i} \not \subset(q=0)$, we see that $\operatorname{dim}_{0}\left(g_{i} Y_{i} \cap(q=0)\right) \leq \operatorname{dim} Y_{i}-1$. Since $(q=0) \backslash\{0\}$ is homogeneous, using Lemma 2 we see that

$$
\operatorname{dim}_{0}\left(g_{1} Y_{1} \cap g_{2} Y_{2} \cap(q=0)\right) \leq\left(\operatorname{dim} Y_{1}-1\right)+\left(\operatorname{dim} Y_{2}-1\right)-(n-1),
$$

as needed.

The above arguments show that our approach gives the best results if the orbits of an action are fully understood. In the most extreme case, we have the following classification. The proof relies on some results of [15], that we recall afterwards.

Proposition 1 Let $X$ be an irreducible, normal variety of dimension $\geq 2$ over a field $k$ and $G$ a connected linear algebraic group acting on $X$. Assume that all orbits have dimension either 0 or $\operatorname{dim} X$.

(1) There is at most 1 orbit of dimension 0.

(2) If char $k=0$ and there is a 0 -dimensional orbit, then $X$ is isomorphic to either an affine or a projective cone over a projective, homogeneous $G$-variety $Y$.

Proof. For (1) we may assume that $k$ is algebraically closed. We may then replace $G$ by its reduction to assume $G$ is smooth. Let $P=\left\{p_{i}\right\}$ be the union of the 0 -dimensional orbits and assume $P$ is nonempty. By Proposition 2 there is a projective $G$-variety $Y$ and a $G$ equivariant, affine, surjective morphism $f: X \backslash P \rightarrow Y$, whose general fiber is 1-dimensional by Lemma 4.

By [24, Theorem 3], there is a normal, $G$-equivariant compactification $\bar{X} \supset X$. Let $Z$ be the normalization of the closure of the graph of $f$ with projections $\pi_{X}$ and $\pi_{Y}$.

Since $G$ acts transitively on $X \backslash P$, it also acts transitively on $Y$, hence $E:=Z \backslash(X \backslash P)$ is a union of $G$-orbits. Thus every fiber of $\pi_{Y}: Z \rightarrow Y$ is a geometrically rational curve and $E$ is a disjoint union of (possibly multiple) sections.

For any $p_{i} \in P$ there is an irreducible component $E_{i} \subset E$ that is contracted by $\pi_{X}$ to $p_{i}$. Let $C \subset Y$ be a general curve, $X_{C}$ the normalization of $\pi_{Y}^{-1}(C)$ and $F_{i} \subset X_{C}$ the preimage of $E_{i}$. Then $X_{C} \rightarrow C$ is a $\mathbb{P}^{1}$-bundle. Note that a $\mathbb{P}^{1}$-bundle over a smooth, projective curve contains at most 1 curve with negative self-intersection, and this curve is a section. Thus $P$ has at most 1 point.

In order to prove (2), we assume from now on that char $k=0$. Then $\pi_{Y}: Z \rightarrow Y$ is a $\mathbb{P}^{1}$-bundle and $E$ consists of 1 or 2 sections.

If $E$ consists of 2 sections, then $Z=\mathbb{P}_{Y}\left(\mathcal{O}_{Y}+L\right)$ for some anti-ample line bundle $L$ on $Y$. Thus $X$ is the affine cone over $\left(Y, L^{-1}\right)$. 
If $E$ consists of 1 section, then $Z=\mathbb{P}_{Y}(E)$ where $E$ is obtained as an extension

$$
0 \rightarrow \mathcal{O}_{Y} \rightarrow E \rightarrow L \rightarrow 0
$$

for some anti-ample line bundle $L$ on $Y$. Now we again use that char $k=0$, hence $-K_{Y}$ is ample and Kodaira's vanishing theorem implies that the extension splits. Thus $X$ is the projective cone over $\left(Y, L^{-1}\right)$.

Remark 1.3. We believe that (2) is not true if char $k \neq 0$, but it should be possible to get a complete description of all cases.

From this we deduce a useful corollary, used in the proof of Corollary 2 above.

Corollary 5 Let $X$ be an irreducible, smooth variety over a field $k$ and $G$ a connected linear algebraic group acting on $X$ with 2 orbits, one of which is a point $x \in X$. Then $X \backslash\{x\} \cong G / H$ where $H$ is connected.

Proof This is clear if $\operatorname{dim} X=1$, so assume that $\operatorname{dim} X \geq 2$.

Let $H_{0} \subset H$ be the identity component. If $H_{0} \neq H$ then $G / H_{0} \rightarrow G / H$ is an étale cover, which extends to an étale cover $\pi: \tilde{X} \rightarrow X$ by purity. The $G$-action on $\tilde{X}$ has an open orbit $\tilde{X} \backslash \pi^{-1}(x)$ and $\pi^{-1}(x)$ is a union of 0 -dimensional orbits. Thus $\operatorname{deg} \pi=1$ by Propositon 1 .

Lemma 4 (cf. [15, p. 443]) Let $X$ be a normal irreducible variety, $P \subset X$ a 0-dimensional subset and $f: X \backslash P \rightarrow Y$ an affine morphism. Then $\operatorname{dim} X \leq \operatorname{dim} Y+1$.

Proof Assume that $\operatorname{dim} X \geq \operatorname{dim} Y+1$. Choose normal compactifications $\bar{X} \supset X, \bar{Y} \supset Y$ and let $Z$ be the normalization of the closure of the graph of $f$ with projections $\pi_{X}$ and $\pi_{Y}$. Note that $\pi_{X}$ cannot contract a whole fiber of $\pi_{Y}$. Thus there is point $\bar{y} \in \bar{Y}$ and an irreducible component $Z_{z} \subset \pi_{Y}^{-1}(\bar{y})$ such that $Z_{z} \cap \pi_{X}^{-1}(P)$ and $Z_{z} \backslash \pi_{X}^{-1}(P)$ are both nonempty. Set $W=\pi_{X}\left(Z_{z}\right)$. Note that $P \cap W \neq \emptyset$, so $W^{\circ}:=W \cap X$ is dense in $W$. Thus $W^{\circ} \cap(X \backslash P)$ is the fiber of $f$, hence affine. By Hartogs's theorem, this implies $\operatorname{dim} W=1$. So the general fiber dimension of $f$ is $\leq 1$.

The following group theoretic result is proved, but not stated, on [15, p. 443]. It does not seem to be well known, so we now state it and give a proof in Paragraph, following suggestions of Brian Conrad and Zhiwei Yun.

Proposition 2 Suppose $X=G / H$ is a homogeneous space for a smooth connected linear algebraic group $G$ over an algebraically closed field $k$. Then, there exists a parabolic subgroup $H \subset P \subset G$ with $P / H$ affine. In particular, there exists a projective variety $Y$ and $a$ surjective and affine map $X \rightarrow Y$.

Remark 3 Knop's proof of Proposition 2 in [15] is slightly different from ours in that he produces a specific choice of $P_{H}$ associated to $H$, whereas our proof merely takes $P$ to be an arbitrary minimal parabolic containing $H$. It is not clear to us what internal property distinguishes it from the other choices.

An interesting aspect is that $P$ is usually not unique and the set of such parabolics has neither a smallest nor a largest element. For example, for

$$
H:=\left(\begin{array}{ll}
* & 0 \\
0 & *
\end{array}\right) \subset \mathrm{SL}_{2}
$$


the maximal choice of $P$ is $\mathrm{SL}_{2}$; the minimal choices are either the upper or the lower triangular matrices. For

$$
H:=\left(\begin{array}{lll}
1 & 0 & * \\
0 & 1 & 0 \\
0 & 0 & 1
\end{array}\right) \subset \mathrm{SL}_{3}
$$

the minimal choice is $B:=$ (upper triangular matrices). The maximal choices are the 2 maximal proper parabolics that contain $B$.

In what follows, for $G$ an algebraic group, we use $R_{u}(G)$ to denote its unipotent radical, the maximal smooth normal connected unipotent subgroup of $G$.

Lemma 5 Let $H$ be a subgroup of a smooth connected reductive group $G$ over a perfect field $k$. Then $H$ is either reductive or contained in a k-parabolic subgroup of $G$.

Proof The key input in this proof is the fact that any smooth connected unipotent subgroup $U$ of a connected linear algebraic group $G$ over a perfect field $k$ is contained in the unipotent radical of a parabolic $k$-subgroup $P \subset G$. This follows from a theorem of Bruhat-Tits, see the "Refined Theorem" in [19].

Applying this to our situation, suppose $H$ is not reductive. We wish to show $H$ is contained in a proper parabolic subgroup. By the above fact, there is some $P \subsetneq G$ with $R_{u}(H) \subset$ $R_{u}(P)$. It follows that $R_{u}(H) \subset H \subset N_{G}\left(R_{u}(H)\right) \subset N_{G}\left(R_{u}(P)\right) \subset N_{G}(P)=P$. Therefore, $H \subset P \subsetneq G$ for $P$ parabolic.

88(Proof of Knop's Proposition 2.) We can write $X=G / H$ for $H \subset G$ a subgroup. Let $P$ denote a minimal parabolic containing $H$. We wish to show $P / H$ is affine. Let $L:=P / R_{u}(P)$ and let $K$ denote the image of $H$ in $L$. Because $P$ was chosen to be a minimal parabolic containing $H, K$ is not contained in any proper parabolic subgroup of $L$. By Lemma $5, K$ is reductive. Let $U:=\operatorname{ker}(H \rightarrow K)$. Then, $U \subset R_{u}(P)$ so $U$ is unipotent. The quotient $R_{u}(P) / U$ is an affine group scheme acting on $P / H$ with quotient $L / K$. It follows that $P / H$ is a principal $R_{u}(P) / U$-bundle over $L / K$, and so to show $P / H$ is affine, it suffices to show $L / K$ is. This follows from the general claim that a quotient of a connected reductive group by a connected reductive subgroup is affine, see [1, Theorem 1.5].

\section{Other applications}

Example 2 (Projective homogeneous spaces) These are of the form $X=G / P$ where $G$ is a semisimple algebraic group and $P \subset G$ a parabolic subgroup. We get a Schubert cell decomposition with a single fixed point $x_{0}$ and an open cell $X^{*} \subset X$. Note that $X^{*}$ is a homogeneous space under the unipotent radical $U \subset P$. The stabilizer of the $U$-action on $X^{*}$ is trivial, hence connected. Thus we get the following.

Claim 2.1. Let $Y_{1}, Y_{2}$ be irreducible, normal varieties and $p_{i}: Y_{i} \rightarrow X$ quasi-finite morphisms. Assume that there is an irreducible component

$$
Z^{*} \subset Y_{1} \times_{X} Y_{2} \times_{X} X^{*} \text { and a point } z_{0}=\left(y_{1}, y_{2}\right) \in Z \subset Y_{1} \times_{X} Y_{2},
$$

such that $\operatorname{dim} Z^{*}=\operatorname{dim} Y_{1}+\operatorname{dim} Y_{2}-\operatorname{dim} X$ and $p_{i}\left(y_{i}\right)=x_{0}$, where $Z$ denotes the closure of $Z^{*}$. Then, for general $\left(g_{1}, g_{2}\right) \in U \times U$, (hece also for general $\left.\left(g_{1}, g_{2}\right) \in G \times G\right)$, the natural map

$$
\pi_{1}\left(g_{1} Y_{1} \times{ }_{X} g_{2} Y_{2}, z_{0}\right) \rightarrow \pi_{1}\left(Y_{1}, y_{1}\right) \times \pi_{1}\left(Y_{2}, y_{2}\right) \text { is }
$$


(a) surjective if char $k=0$ and

(b) surjective on finite quotients if char $k>0$.

Note that we could consider instead the $P$-action, which has a usually larger open orbit $X^{\circ} \supset X^{*}$. This gives the following variant.

Claim 2.2. Using the above notation, assume that

$$
\operatorname{dim} Y_{1}+\operatorname{dim} Y_{2}>\operatorname{dim} X+\operatorname{dim}\left(X \backslash X^{\circ}\right) .
$$

Then the natural map

$$
\pi_{1}\left(g_{1} Y_{1} \times{ }_{X} g_{2} Y_{2}, z_{0}\right) \rightarrow \pi_{1}\left(Y_{1}, y_{1}\right) \times \pi_{1}\left(Y_{2}, y_{2}\right) \text { is }
$$

(b) surjective if char $k=0$, and

(c) surjective on finite quotients if char $k>0$,

for general $\left(g_{1}, g_{2}\right) \in P \times P$.

Proof For dimension reasons there is an irreducible component $Z^{\circ} \subset Y_{1} \times_{X} Y_{2} \times_{X} X^{\circ}$ that contains a good limit point $z_{0}$. Next we use the $P$-action to see that $z_{0}$ is also a good limit point in $g Z^{\circ} \times{ }_{X} X^{*}$ for general $g \in P$. Now we can apply (1).

Results of this type have been considered in [5,9,10,22]. Our bound (2.2.a) is optimal in some cases, but weaker in several of them. Using the full $G$-action, as in the above articles, leads to further improvements, but we did not find a natural way to recover the bounds of $[5,9]$ in all cases.

Example 3 (Prehomogeneous vector spaces) A prehomogeneous vector space is a pair $\left(G, V^{n}\right)$ where $V^{n}$ is a $k$-vector space of dimension $n$ and $G \subset \mathrm{GL}_{n}$ is a connected subgroup that has a dense orbit $W \subset V^{n}$. See [13] for an introduction and detailed classification.

The infinite series of irreducible ones all have connected generic stabilizers. Using the original Sato-Kimura numbering as in [13], the basic examples are built from

(1) $\left(\mathrm{SL}_{n}, V^{n}\right)$,

(2) $\left(\mathrm{SL}_{n}, \mathrm{Sym}^{2} V^{n}\right)$,

(3) $\left(\mathrm{SL}_{n}, \wedge^{2} V^{n}\right)$,

(13) $\left(\mathrm{Sp}_{n}, V^{2 n}\right)$,

(15) $\left(\mathrm{O}_{n}, V^{n}\right)$

These lead to further examples by enlarging the group to contain the scalars or replacing $\left(G, V^{n}\right)$ with $\left(G \times \mathrm{SL}_{m}, V^{n} \otimes V^{m}\right)$ for certain values of $m$.

Most of the sporadic examples either have disconnected generic stabilizer or the connectedness is not known. A nice example is $\left(\mathrm{E}_{6} \cdot \mathbb{G}_{m}, V^{27}\right)$, which is no. $27^{1}$ on the list. The connected component of the generic stabilizer is $\mathrm{F}_{4}$. Since $\mathrm{F}_{4}$ has no outer automorphisms, the stabilizer is $\mathrm{F}_{4}$, hence connected. See also $[17,23,26,27]$ for several other examples.

\section{Counterexamples}

Example 4 Start with $\left(\mathrm{GL}_{3}, \mathbb{A}_{x y z}^{3}\right)$ and let $Y_{1}, Y_{2} \subset \mathbb{A}^{3}$ be cones with vertex 0 . Then $g_{1} Y_{1} \cap$ $g_{2} Y_{2}$ conists of $\operatorname{deg} Y_{1} \cdot \operatorname{deg} Y_{2}$ lines for general $g_{1}, g_{2} \in \mathrm{GL}_{3}$. Thus, so long as $Y_{1}$ and $Y_{2}$ are not both planes, $\left(g_{1} Y_{1} \cap g_{2} Y_{2}\right)(0)=g_{1} Y_{1} \cap g_{2} Y_{2}$ is reducible and the origin is a good limit point.

$127=27$ is a coincidence. 
Example 5 Consider $\left(\mathbb{G}_{m}^{3}, \mathbb{A}_{x y z}^{3}\right)$ and let let $Y_{1}, Y_{2} \subset \mathbb{A}^{3}$ be surfaces that contain the $z$-axis. Usually $g_{1} Y_{1} \cap g_{2} Y_{2}$ is reducible, having both moving and fixed irreducible components for $g_{1}, g_{2} \in \mathbb{G}_{m}^{3}$.

If $Y_{1}, Y_{2}$ intersect transversally at the origin then the origin is not a good limit point, even though $g_{1} Y_{1} \cap g_{2} Y_{2}$ may have non-empty intersection with the dense open orbit. For example, this happens for $Y_{1}=\left(y=x^{2}\right), Y_{2}=\left(x=y^{2}\right)$.

In any case, the origin is a good limit point if $Y_{1} \cap Y_{2}$ has an irreducible component $Z$ that passes through the origin but is not contained in a coordinate hyperplane.

Example 6 Suppose $k$ has characteristic 0 (or at least has characteristic not equal to 2). Let $X \subset \mathbb{P}^{4}$ be the projective cone over a smooth quadric surface with vertex $x_{0}$. Let $G \subset \operatorname{Aut}(X)$ be the identity component. Then, $G$ acts with 2 orbits: $\left\{x_{0}\right\}$ and $X \backslash\left\{x_{0}\right\}$. Let $Y_{1}$ be a 2-plane contained in $X$ and containing $x_{0}$. We claim that there is a divisor $Y_{2}$ with $x_{0} \in Y_{2} \subset X$ such that $g_{1} Y_{1}^{n} \times{ }_{X} g_{2} Y_{2}^{n}$ is the union of a curve and a point for general $\left(g_{1}, g_{2}\right) \in G \times G$. Thus one preimage of $x_{0}$ is a good limit point, the other is not.

The computation is local at $x_{0}$, thus we choose affine coordinates such that $X=(x y-u v=$ $0)$. We can then choose $Y_{1}=(x=u=0)$. We choose $Y_{2}$ to be the complete intersection $\left(x y-u v=4 u-(x-y)^{2}=0\right)$. We can eliminate $u$ to get

$$
Y_{2} \cong\left(4 x y-(x-y)^{2} v=0\right) \subset \mathbb{A}^{3} .
$$

This is an irreducible hypersurface, but

$$
4 x y-(x-y)^{2} v=(x+y+\sqrt{1+v}(x-y))(x+y-\sqrt{1+v}(x-y))
$$

shows that it is non-normal along $(x=y=0)$ and $Y_{2}^{n}$ has 2 points over the origin. We will next show that only one of them is a good limit point.

A typical translate of $Y_{1}$ by $G$ is $\left(x-c^{-1} v=y-c u=0\right)$. Add these to the equations

$$
x+y \pm \sqrt{1+v}(x-y)=4 u-(x-y)^{2}=0
$$

and eliminate $u$ and $v$ to get

$$
x+y \pm \sqrt{1+c x}(x-y)=0 \text { and } \quad(6.1) 4 c^{-1} y-(x-y)^{2}=0 .
$$

Here (6.2) defines a curve whose tangent line at the origin is $y=0$. With + sign, (6.1) defines a curve whose tangent line at the origin is $x=0$. So we get an isolated intersection point at the origin. Finally,

$$
y=x \frac{\sqrt{1+c x}-1}{\sqrt{1+c x}+1}
$$

satisfies both (6.1) with a + sign and (6.2).

Example 7 Suppose $k$ has characteristic 0 . For $X=\mathbb{P}^{n} \times \mathbb{P}^{n}$, consider $G=\mathrm{PGL}_{n+1} \times$ $\mathrm{PGL}_{n+1}, Y_{1}=\mathbb{P}^{n} \times C$, and $Y_{2}=\mathbb{P}^{n} \times H$, where $C \subset \mathbb{P}^{n}$ is a smooth projective curve of positive genus and $H \subset \mathbb{P}^{n}$ a hypersurface. Then $\operatorname{dim} Y_{1}+\operatorname{dim} Y_{2}=\operatorname{dim} X+n$.

For general $\left(g_{1}, g_{2}\right) \in G \times G$,

$$
g_{1} Y_{1} \cap g_{1} Y_{2}=\mathbb{P}^{n} \times(\operatorname{deg} C \cdot \operatorname{deg} H \text { points }) .
$$

Thus, the intersection is disconnected and its conected components are simply connected. So, they do not contribute to the fundamental group of $C$ and hence $\pi_{1}\left(g_{1} Y_{1} \cap g_{2} Y_{2}, z_{0}\right) \rightarrow$ $\pi_{1}\left(Y_{1}, y_{1}\right)$ has infinite index for any basepoint $z_{0} \in g_{1} Y_{1} \cap g_{2} Y_{2}$. 
Example 8 (Variant of [10, Example, p. 634]) Suppose $k$ has characteristic 0. In $X=$ $\operatorname{Gr}\left(\mathbb{P}^{1}, \mathbb{P}^{n}\right)$, take $G=\mathrm{PGL}_{n+1}$ and consider

$$
\begin{aligned}
Y_{1} & =(\text { lines through a point }) \cong \mathbb{P}^{n-1}, \\
Z_{L} & =\left(\text { lines in a hyperplane } L \subset \mathbb{P}^{n}\right) \cong \operatorname{Gr}\left(\mathbb{P}^{1}, \mathbb{P}^{n-1}\right), \text { and } \\
Y_{2} & =\cup_{c \in C} Z_{L_{c}},
\end{aligned}
$$

where $C \subset \check{\mathbb{P}}^{n}$ is a smooth projective curve of positive genus parametrizing hyperplanes $\left\{L_{c}: c \in C\right\}$. Then

$$
\operatorname{dim} Y_{1}+\operatorname{dim} Y_{2}=\operatorname{dim} X+n-2 .
$$

The key property is that $g_{1} Y_{1} \cap g_{2} Z_{L}$ is either empty or is isomorphic to $\mathbb{P}^{n-2}$. Thus, for general $\left(g_{1}, g_{2}\right) \in G \times G, g_{1} Y_{1} \cap g_{2} Y_{2}$ is reducible and its connected components are isomorphic to $\mathbb{P}^{n-2}$. So again they do not contribute to the fundamental group of $C$.

Example 9 Let $k$ be a field of positive characteristic, take $X=\mathbb{P}^{2}$ with the standard action of $G=$ PGL $_{3}$. Let $Y_{1} \subset \mathbb{P}^{2}$ be a line and let $Y_{2} \subset \mathbb{P}^{2}$ be the complement of a line. A general translate of an intersection of $Y_{1}$ with $Y_{2}$ is isomorphic to $\mathbb{A}^{1}$, and the map $g_{1} Y_{1} \cap g_{2} Y_{2} \rightarrow Y_{2}$ is identified with the inclusion of a line $\mathbb{A}^{1} \rightarrow \mathbb{A}^{2}$. Hence, after applying an automorphism, we may assume it is given by $\operatorname{Spec} k[x, y] /(y) \rightarrow \operatorname{Spec} k[x, y]$. This is not surjective on fundamental groups because the pullback of the connected finite étale Artin-Schreier cover $W:=\operatorname{Spec} k[x, y, t] /\left(t^{p}-t-y\right) \rightarrow \operatorname{Spec} k[x, y]$ is not connected.

\section{Examples with disconnected stabilizers}

We conclude by giving examples showing that $\pi_{1}\left(g_{1} Y_{1} \times{ }_{X} g_{2} Y_{2}, z_{0}\right) \rightarrow \pi_{1}\left(Y_{1}, y_{1}\right) \times$ $\pi_{1}\left(Y_{2}, y_{2}\right)$ may be non-surjective when $H$ is disconnected in the setting of Theorem 2 . For the remainder of the paper, we assume $k$ has characteristic 0 .

Example 10 If $X$ as in Theorem 2 has nontrivial fundamental group, we can take $Y_{1}=Y_{2}=$ $X$, and the resulting map $\pi_{1}\left(X, x_{0}\right) \rightarrow \pi_{1}\left(X, x_{0}\right) \times \pi_{1}\left(X, x_{0}\right)$ will fail to be surjective. By applying Theorem 2 , we conclude that $H$ must be disconnected, and have at least $\pi_{1}\left(X, x_{0}\right)$ components.

For a concrete example of such a variety, take $X$ to be the moduli space parametrizing unordered pairs of distinct points in $\mathbb{P}^{2}$. Then $X$ is homogeneous under $\mathrm{PGL}_{3}$. The open orbit in $X \times X$ is formed by those $\left(\left\{p_{1}, p_{2}\right\},\left\{p_{1}^{\prime}, p_{2}^{\prime}\right\}\right)$ for which no three points are on a line. The stabilizer is $\mathbb{Z} / 2 \times \mathbb{Z} / 2$. The space parametrizing ordered pairs of distinct points in $\mathbb{P}^{2}$ is the universal cover of $X$. Thus $\pi_{1}(X) \cong \mathbb{Z} / 2$.

We conclude by giving a somewhat more involved example where $H$ is disconnected, but nevertheless $\pi_{1}\left(X, x_{0}\right)=1$.

Example 11 (Constructing $X$ and $G$ ) Let $X$ be the moduli space of smooth plane conics in $\mathbb{P}^{4}$ over $\mathbb{C}$. The group GL5 acts transitively on $X$ via its action on $\mathbb{P}^{4}$.

Choose coordinates $x_{0}, \ldots, x_{4}$ and a reference conic $C_{0}=\left(x_{0}^{2}+x_{1}^{2}+x_{3}^{2}=x_{3}=x_{4}=0\right)$. The stablizer of $C_{0}$ is the set of matrices

$$
\tilde{G}:=\left\{\left(\begin{array}{cc}
\mathrm{GO}_{3} & * \\
0 & \mathrm{GL}_{2}
\end{array}\right)\right\} \subset \mathrm{GL}_{5} .
$$


Thus $\tilde{G}$ is connected and so is $\tilde{G} \cap \mathrm{SL}_{5}$ as shown by the retraction

$$
\left(\begin{array}{cc}
A_{3} & B \\
0 & A_{2}
\end{array}\right) \mapsto\left(\begin{array}{cc}
d^{-1} A_{3} & B \\
0 & d A_{2}
\end{array}\right) \text { where } d=\operatorname{det} A_{3} \cdot \operatorname{det} A_{2} .
$$

Thus $X \cong \mathrm{SL}_{5} /\left(\tilde{G} \cap \mathrm{SL}_{5}\right)$ is simply connected. Since we prefer faithful actions, our group $G \subset \mathrm{PGL}_{5}$ is the image of $\tilde{G}$.

For $Z \subset \mathbb{P}^{4}$, let $\langle Z\rangle$ denote the linear span of $Z$ in $\mathbb{P}^{4}$. Observe that $G$ has a dense orbit $X^{\circ} \subset X$, consisting of those conics $C_{1}$ so that $\left\langle C_{0}\right\rangle \cap\left\langle C_{1}\right\rangle$ is a point $p$ and neither of the $C_{i}$ contains $p$.

If $C_{1}=\left(x_{0}=x_{1}=x_{2}^{2}+x_{3}^{2}+x_{4}^{2}=0\right)$ then the stabilizer of the ordered pair $\left(C_{0}, C_{1}\right)$ is the set of matrices

$$
H:=\left\{\left(\begin{array}{ccc}
\mathrm{O}_{2} & 0 & 0 \\
0 & 1 & 0 \\
0 & 0 & \mathrm{O}_{2}
\end{array}\right)\right\} \subset \text { PGL }_{5} .
$$

Thus $H$ has 4 connected components, which can be geometrically described as follows. Set $p:=\left\langle C_{0}\right\rangle \cap\left\langle C_{1}\right\rangle$. From $p$ one can draw two distinct tangent lines to each $C_{i}$. Let these tangent lines be $\left\{T_{0}^{0}, T_{0}^{1}\right\}$ and $\left\{T_{1}^{0}, T_{1}^{1}\right\}$. The $H$ action permutes these lines, giving a surjection $H \rightarrow \mathbb{Z} / 2 \times \mathbb{Z} / 2$.

Example 12 (Constructing $Y_{1}$ and $Y_{2}$ ) Continuing Example 11, we next construct the subvarieties $Y_{1}$ and $Y_{2} \subset X$.

Choose a point $q \in\left\langle C_{0}\right\rangle \backslash C_{0}$ and let $Y_{1}:=Y_{1}(q) \subset X$ be the set of those conics $C$ for which $q \in\langle C\rangle \backslash C$. Then $Y_{1}$ is a smooth, locally closed subvariety of dimension 9 in $X$.

To construct $Y_{2}$, fix 2 distinct points $x_{1}, x_{2} \in C_{0}$ and general 2-planes $S_{i}$ such that $x_{i} \in S_{i}$. Let $Y_{2}:=Y_{2}\left(S_{1}, S_{2}\right) \subset X$ be the set of conics $C$ with the following two properties.

(1) We have that $c_{i}:=C \cap S_{i}$ is a single point and $c_{1} \neq c_{2}$.

(2) Let $\tau(C) \in\langle C\rangle \backslash C$ denote the intersection point of the lines tangent to $C$ at the $c_{i}$. Then $\tau(C) \in\left\langle C_{0}\right\rangle \backslash C_{0}$.

Given distinct $c_{i} \in S_{i}$ and $p \in\left\langle C_{0}\right\rangle \backslash C_{0}$, the set of such conics for $c_{i}=C \cap S_{i}$ and $\tau(C)=p$ is a principal $\mathbb{G}_{m}$-bundle whenever $c_{1}, c_{2}$, and $p$ are not collinear. Indeed, the conics in $\mathbb{P}^{2}$ that pass through $(1: 0: 0),(0: 1: 0)$ which have tangent lines at those points intersecting at $(0: 0: 1)$ are precisely the hyperbolas $x_{1} x_{2}=\lambda x_{0}^{2}$ for $\lambda \in \mathbb{G}_{m}(\mathbb{C})$. Thus $Y_{2}$ is a smooth, locally closed subvariety of dimension 7 in $X$.

The intersection $Z:=Y_{1} \cap Y_{2}$ consists of those conics in $Y_{2}$ for which $\tau(C)=q$. Thus $Z$ is a smooth, locally closed subvariety of dimension 5 in $X$.

Proposition 3 We use the notation of Examples 11-12. For general $\left(g_{1}, g_{2}\right) \in G \times G$, $g_{1} Y_{1} \cap g_{2} Y_{2}$ is irreducible and the map

$$
\pi_{1}\left(g_{1} Y_{1} \cap g_{2} Y_{2},\left[C_{0}\right]\right) \rightarrow \pi_{1}\left(Y_{1},\left[C_{0}\right]\right) \text { has index } 4 .
$$

Proof Note first that the group action sends $Y_{1}(q)$ to $Y_{1}\left(g_{1} q\right)$ and $Y_{2}\left(S_{1}, S_{2}\right)$ to $Y_{2}\left(g_{2} S_{1}, g_{2} S_{2}\right)$. Thus, letting $Z=Y_{1} \cap Y_{2}$, it is enough to show that

$$
\pi_{1}\left(Z,\left[C_{0}\right]\right) \rightarrow \pi_{1}\left(Y_{1},\left[C_{0}\right]\right) \times \pi_{1}\left(Y_{2},\left[C_{0}\right]\right) \text { has index } 4 .
$$

The index is at most 4 by Theorem 2 because $H$ has 4 connected components, as shown in Example 11. We now show the index is at least 4 . Note that $Y_{1}$ has a connected degree 2 finite étale cover $\widetilde{Y}_{1} \rightarrow Y_{1}$ parametrizing pairs $(C, c)$ where $C \in Y_{1}$ and $c \in C$ is one of the 
2 points of $C$ whose tangent line passes through $q$. Similarly, $Y_{2}$ has a connected degree 2 finite étale cover parametrizing pairs $(C, d)$ where $d$ is one of the two points of $C_{0}$ whose tangent line passes through $\tau(C)$. Let $q_{1}$ and $q_{2}$ denote the two points of $C_{0}$ whose tangent lines pass through $q$. Then, the restriction of the cover $\widetilde{Y}_{1} \times \widetilde{Y}_{2}$ to $Z$ splits into the 4 connected components

$$
\widetilde{Z}_{i, j}:=\left\{\left(C, C \cap S_{i}, q_{j}\right)\right\} \subset Z \times \times_{Y_{1} \times Y_{2}}\left(\widetilde{Y}_{1} \times \widetilde{Y}_{2}\right) \quad \text { for } \quad 1 \leq i, j \leq 2 .
$$

Acknowledgements We thank Brian Conrad and Zhiwei Yun for explaining the proof of Proposition 2, Robert Lazarsfeld for pointing out Corollary 1, Laurent Manivel, Anand Patel, and Arpon Raksit for helpful conversations, and Akihiko Yukie for long comments and references on prehomogeneous vector spaces. We also thank François Charles, Dougal Davis, Laurent Moret-Bailly, Wenhao Ou, Eric Riedl, Zev Rosengarten, Jason Starr, Ashvin Swaminathan, and Ravi Vakil for helpful correspondence on an earlier version of this article. JK was supported by the NSF under grant number DMS-1901855 and AL by the National Science Foundation Graduate Research Fellowship Program under Grant No. DGE-1656518 as well as through the program "Oberwolfach Research Fellows" by the Mathematisches Forschungsinstitut Oberwolfach in 2021.

Funding Open Access funding enabled and organized by Projekt DEAL.

\section{Declarations}

Conflict of interest Statement On behalf of all authors, the corresponding author states that there is no conflict of interest.

Open Access This article is licensed under a Creative Commons Attribution 4.0 International License, which permits use, sharing, adaptation, distribution and reproduction in any medium or format, as long as you give appropriate credit to the original author(s) and the source, provide a link to the Creative Commons licence, and indicate if changes were made. The images or other third party material in this article are included in the article's Creative Commons licence, unless indicated otherwise in a credit line to the material. If material is not included in the article's Creative Commons licence and your intended use is not permitted by statutory regulation or exceeds the permitted use, you will need to obtain permission directly from the copyright holder. To view a copy of this licence, visit http://creativecommons.org/licenses/by/4.0/.

\section{References}

1. Borel, A.: On affine algebraic homogeneous spaces, Arch. Math. (Basel) 45 (1985), no. 1, 74-78. MR 799451

2. Catanese, F.: Topological methods in algebraic geometry, Colloquium De Giorgi 2013 and 2014, Colloquia, vol. 5, Ed. Norm., Pisa, 2014, pp. 37-77. MR 3379178

3. Catanese, F.: Topological methods in moduli theory, Bull. Math. Sci. 5 (2015), no. 3, 287-449. MR 3404712

4. Deligne, P.: Le groupe fondamental du complément d'une courbe plane n'ayant que des points doubles ordinaires est abélien (d'après W. Fulton), Bourbaki Seminar, Vol. 1979/80, Lecture Notes in Math., vol. 842, Springer, Berlin-New York, 1981, pp. 1-10. MR 636513

5. Faltings, G.: Formale Geometrie und homogene Räume, Invent. Math. 64 (1981), no. 1, 123-165. MR 621773

6. Fulton, W., Hansen, J.: A connectedness theorem for projective varieties, with applications to intersections and singularities of mappings, Ann. of Math. (2) 110 (1979), no. 1, 159-166. MR 541334

7. Fulton, W., Lazarsfeld, R.: Connectivity and its applications in algebraic geometry, Algebraic geometry (Chicago, Ill., 1980), Lecture Notes in Math., vol. 862, Springer, Berlin-New York, 1981, pp. 26-92. MR 644817

8. Goresky, M., MacPherson, R.: Stratified Morse theory, Ergebnisse der Mathematik und ihrer Grenzgebiete (3) [Results in Mathematics and Related Areas (3)], vol. 14, Springer-Verlag, Berlin, 1988. MR 932724

9. Goldstein, N.: Ampleness and connectedness in complex G/P, Trans. Amer. Math. Soc. 274 (1982), no. 1, 361-373. MR 670938 
10. Hansen, J.: A connectedness theorem for flagmanifolds and Grassmannians, Amer. J. Math. 105 (1983), no. 3, 633-639. MR 704218

11. Hironaka, H.: Stratification and flatness, Real and complex singularities (Proc. Ninth Nordic Summer School/NAVF Sympos. Math., Oslo, 1976), 1977, pp. 199-265. MR 0499286

12. Jouanolou, J.-P.: Théorèmes de Bertini et applications, Progress in Mathematics, vol. 42, Birkhäuser Boston, Inc., Boston, MA, 1983. MR 725671

13. Kimura, T.: Introduction to prehomogeneous vector spaces, Translations of Mathematical Monographs, vol. 215, American Mathematical Society, Providence, RI, 2003, Translated from the 1998 Japanese original by Makoto Nagura and Tsuyoshi Niitani and revised by the author. MR 1944442

14. Kleiman, S. L.: The transversality of a general translate, Compositio Math. 28 (1974), 287-297. MR 360616

15. Knop, F.: Mehrfach transitive Operationen algebraischer Gruppen, Arch. Math. (Basel) 41 (1983), no. 5, 438-446. MR 731620

16. Kramer, L.: Two-transitive Lie groups, J. Reine Angew. Math. 563 (2003), 83-113. MR 2009240

17. Kato, R., Yukie, A.: Rational orbits of the space of pairs of exceptional Jordan algebras, J. Number Theory 189 (2018), 304-352. MR 3788652

18. Lazarsfeld, R.: Positivity in algebraic geometry. I, Ergebnisse der Mathematik und ihrer Grenzgebiete. 3. Folge. A Series of Modern Surveys in Mathematics [Results in Mathematics and Related Areas. 3rd Series. A Series of Modern Surveys in Mathematics], vol. 48, Springer-Verlag, Berlin, 2004, Classical setting: line bundles and linear series. MR 2095471

19. User 22479 MathOverflow, Homomorphism into reductive groups, MathOverflow, 2012, https:// mathoverflow.net/q/104266 (version: 2012-08-09)

20. Mori, S.: Projective manifolds with ample tangent bundles, Ann. of Math. (2) 110 (1979), no. 3, 593-606. MR 554387

21. Sommese, A. J.: Submanifolds of Abelian varieties, Math. Ann. 233 (1978), no. 3, 229-256. MR 466647

22. Sommese, A. J.: Complex subspaces of homogeneous complex manifolds. I. Transplanting theorems, Duke Math. J. 46 (1979), no. 3, 527-548. MR 544244

23. Springer, T. A..: Some groups of type $E_{7}$, Nagoya Math. J. 182 (2006), 259-284. MR 2235344

24. Sumihiro, H.: Equivariant completion, J. Math. Kyoto Univ. 14 (1974), 1-28. MR 337963

25. Tits, J.: Sur certaines classes d'espaces homogènes de groupes de Lie, Acad. Roy. Belg. Cl. Sci. Mém. Coll. in $8^{\circ} \mathbf{2 9}$ (1955), no. 3, 268. MR 76286

26. Wright, D. J. ., Yukie, A.: Prehomogeneous vector spaces and field extensions, Invent. Math. 110 (1992), no. 2, 283-314. MR 1185585

27. Yukie, A.: Prehomogeneous vector spaces and field extensions. III, J. Number Theory 67 (1997), no. 1, 115-137. MR 1485429

Publisher's Note Springer Nature remains neutral with regard to jurisdictional claims in published maps and institutional affiliations. 